\title{
Masking of motion by broadband and filtered directional noise
}

\author{
KARLENE BALL and ROBERT SEKULER \\ Cresap Neuroscience Laboratory, Northwestern University, Evanston, Illinois 60201
}

\begin{abstract}
Observers detected unidirectional motion of isotropic dot patterns following exposure to (1) broadband noise containing all directions of motion, or (2) noise from which certain sets of direction had been filtered. The amount of masking varied with the set of directions filtered from the noise, yielding broad directional tuning functions. The breadth of an observers's tuning function corresponded to the precision with which the observer could recognize small differences in direction of motion.
\end{abstract}

Many studies of sensory function require an observer to detect signals in the presence of noise injected into his sensory system by the experimenter. With properly controlled variation in the noise's characteristics, this technique assumes great analytic power. For example, in audition, Fletcher (1940) developed the critical band concept from his demonstration that only those components of broadband noise which were spectrally proximate to the frequency of some test tone were effective in masking that tone. More recently, Patterson (1976) extended Fletcher's approach by centering a notch in the broadband noise spectrum on the signal tone frequency and measuring the tone's threshold as a function of notch width. Armed with certain reasonable assumptions, Patterson was able to refine previous estimates of the frequency-selectivity of auditory mechanisms responsible for the detection of various test frequencies.

Since this approach has such proven power, we decided to apply it to the study of visual, directionally selective mechanisms. Our basic analytic tool was broadband visual noise composed either of all directions of motion or of some limited set of directions. We compared the ability of these two types of noise to mask or reduce the visibility of motion in a particular test direction. This allowed us to define the directional selectivity of the visual mechanisms which detected the test motion.

Recent human psychophysical experiments converge on a unified model of motion detection in which mechanisms, each sensitive to a limited range of directions, play a key role. The psychophysical experiments contributing to the model have used a

This research was supported by Grant BNS77-15858 from the National Science Foundation. We thank all our conscientious observers, particularly David Ball. We also thank Randolph Blake for most helpful suggestions on this paper. Requests for reprints should be sent to Robert Sekuler, Department of Psychology, Cresap Neuroscience Laboratory, Northwestern University, Evanston, Illinois 60201 . variety of techniques, including selective adaptation (Sekuler \& Ganz, 1963), subthreshold summation (Levinson \& Sekuler, 1975), motion aftereffects (Keck, Pallela, \& Pantle, 1976), and others. Since all these studies have used one-dimensional grating patterns, effects associated with the stimulus's orientation have been inextricably bound up with responses to stimulus movement per se. Consequently, these studies cannot give a complete description of the direction selectivity of the mechanisms under examination. To isolate responses to motion independent of contour orientation, we have used stimuli with equal energy along all axes of the visual field. These isotropic stimuli are used in forward masking experiments designed to yield a description of the twodimensional sensitivity profiles of human motionsensitive mechanisms.

Our work also differs from earlier studies of direction selectivity by our use of reaction time as a dependent variable; this was motivated, in part, by our long-term goal of explicating perception of motion under conditions more closely approximating the everyday situation. Since there is reasonable doubt that extrapolations can be made from studies of threshold detection performance to suprathreshold levels (Sekuler, 1975), we decided to use a direct measure of suprathreshold performance itself, speed of response to motion onset. Our experiments use reaction time as an index of the observer's sensitivity to motion. Implicit in this use are a number of assumptions about reaction times and the observer's strategy; we shall discuss those assumptions later in this paper.

Briefly, here is the rationale for our forwardmasking procedure. As indicated before, we assume that direction analyzing mechanisms exist at some stage in the visual system. Each mechanism may be likened to a filter tuned to a characteristic range of directions. Our masking noise, depending upon its directional components, may be injected into the directionally selective filter whose response signals 
the presence of some test direction. As would be true for any real system with a temporal impulse response of greater than zero duration, we assume the effect of the visual noise outlasts the noise itself, lowering the effective signal/noise ratio associated with certain subsequently presented moving targets. This is the forward-masking effect we set out to examine.

\section{GENERAL METHOD}

Stimuli were patterns of isotropic random dots presented on an $\mathrm{X}-\mathrm{Y}$ display under computer control. The isotropy of the dot pattern was assessed by two-dimensional numerical Fourier analysis and by inspection of the pattern's optical transform (Lipson, 1972). The display tube was illuminated by a veiling light to a level of $1.7 \mathrm{~cd} / \mathrm{m}^{2}$; the incremental luminance of each dot was $84 \mathrm{~cd} / \mathrm{m}^{2}$. While sitting with head supported by a chinrest, the observer viewed the display binocularly from a distance of $57 \mathrm{~cm}$. About 400 dots of the pattern were visible at any one moment within an $8^{\circ}$-diam aperture. In each display frame $(33 \mathrm{msec})$, the computer plotted a total of 512 dots on the cathode ray tube (CRT).

Every trial began with a random foreperiod distributed uniformly over the interval of 2.0-3.5 sec. During this foreperiod, the dots appeared as a stationary pattern. Then, without warning, all the dots began to move uniformly, along parallel paths at $5.3^{\circ} / \mathrm{sec}$.

The spatial pattern was created by a random-number-generating algorithm which produced a set of 512 pairs of random numbers scaled appropriately and stored in a table. The computer cycled through the table fetching the members of each random-number pair. The numbers were loaded into two digital-to-analog converters (DACs), one for the CRT beam $X$ position and the other for its Y position. When both DACs were loaded and the beam correctly positioned, the point on the CRT screen was intensified.

The following algorithm created movement in one direction for some period. Let $\mathrm{V}$ be the desired velocity (in degrees per second) and $f$ the frame-rate of the display (the number of display frames per second; in each frame, all 512 dots would be presented). Finally, let $\theta$ be the direction in which movement is desired and $X_{i}$ and $Y_{i}$ the $X, Y$ coordinates for the $i^{\text {th }}$ point.

In each display frame, the computer calculated $X_{i}^{\prime}=X_{i}+$ $(V / f) \cos (\theta)$, and $Y^{\prime}{ }_{i}=Y_{i}+(V / f)$ sine $(\theta)$. The newly calculated $X^{\prime}{ }_{i}$ and $Y^{\prime}{ }_{i}$ were loaded into the appropriate DACs, the beam was positioned, and the spot intensified. The values $X^{\prime}{ }_{i}$ and $Y^{\prime}{ }_{i}$ were then substituted for $X_{i}$ and $Y_{i}$ in the stored table; this substitution caused the motion to cumulate from one frame to the next. Good, compelling motion was achieved at frame rates of $20 \mathrm{~Hz}$ or above.

On each trial, the movement continued until the observer depressed a telegraph key. The pattern then disappeared and the display was blanked until the next trial $(4-5 \mathrm{sec})$. The edges of the display were functionally connected so that a dot moving off one side would reappear a moment later on the opposite side. This gave the display the appearance of an infinite textured surface moving continuously behind the aperture. The interval between onset of pattern movement and the subject's response, reaction time, was measured to the nearest millisecond. To minimize the potentially potent effects of the observer's uncertainty about direction of motion (Sekuler \& Ball, 1977), the first five trials of each block were discarded before data analysis.

\section{EXPERIMENT 1}

Though our stimuli were isotropic, it was possible that observers' sensitivity might not be. For example, stationary gratings of either horizontal or vertical orientation are usually more easily seen than gratings of other orientations (Appelle, 1972). Since some of our later experiments required knowledge of the isotropy of motion sensitivity, our first experiment was designed to compare the observers' sensitivity to motion in various directions.

\section{Method}

Reaction times were measured for 16 different directions of motion: $0^{\circ}, 22.5^{\circ}, 45^{\circ}, 67.5^{\circ}, 90^{\circ}, 112.5^{\circ}, 135^{\circ}, 157.5^{\circ}, 180^{\circ}$, $202.5^{\circ}, 225^{\circ}, 247.5^{\circ}, 270^{\circ}, 292.5^{\circ}, 315^{\circ}$, and $337.5^{\circ}$, where $90^{\circ}$ is motion from bottom to top. A session consisted of 16 blocks of 30 trials each; within 1 block, the direction of motion was constant. A 1-min rest was allowed after each 30-trial block. Three observers (K.B., D.B., and S.L.) each served in four sessions, with the order of the blocks of test directions separately randomized for each observer. All observers had normal or corrected-to-normal vision and were inexperienced for this task.

\section{Results}

Reaction times shorter than $100 \mathrm{msec}$ or longer than $600 \mathrm{msec}$ were discarded from the analysis. Anticipatory responses, less than $100 \mathrm{msec}$, occurred on fewer than $2 \%$ of all trials, and the frequency of anticipations was not related to the test-motion direction. The geometric mean of the remaining reaction times was computed for each observer, session, and test direction. The mean reaction times for all three observers are shown in Figure 1.

The reaction time variance was analyzed by computing the data's projection onto a series of vectors representing the mean and first four sine and cosine terms of a Fourier series: sine $F$, cosine $F$, sine $2 F, \ldots$, cosine 4F (Sekuler \& Armstrong, 1978). Each of

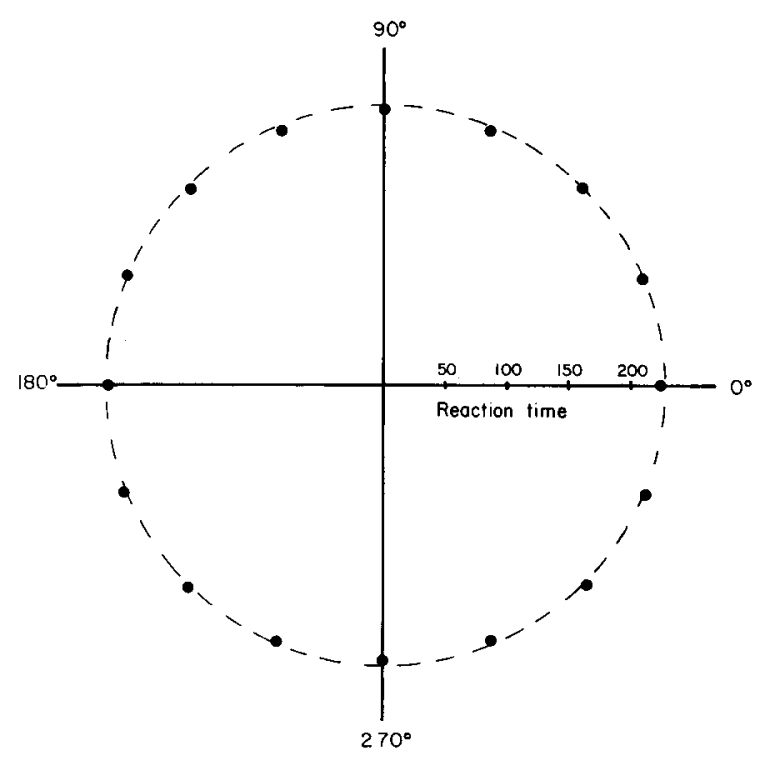

Figure 1. Mean reaction times in milliseconds for motion in each of 16 different directions. The grand mean, 227 msec, for three observers is indicated with a dotted line. 
these Fourier series terms, plus one representing a linear change in reaction time over sessions, had $\mathrm{df}=1$ in the analysis of variance (ANOVA).

The ANOVA revealed a significant decrease in reaction time over sessions $[\mathrm{F}(3,6)=27.74, \mathrm{p}<.001]$; this decrease amounted to a $4 \%$ change. Of the Fourier series terms, only that associated with the mean was significant $[\mathrm{F}(1,48)=9804.6, \mathrm{p}<.001]$. This one term, which reflects the circularity of the data plotted in Figure 1, accounts for $99 \%$ of the variance in our data. Since neither sine $F$ nor cosine $F$ terms approached significance, our data are not only circular on polar coordinates, but the origin of the best fitting circle also is not significantly different from the origin of the polar coordinate system (Sekuler \& Armstrong, 1978). Had our data contained the types of anisotropy characteristic of the oblique effect (Appelle, 1972), we would have expected longer reaction times for movement in the oblique directions: $45^{\circ}, 135^{\circ}, 225^{\circ}$, and $315^{\circ}$. The absence of significant sine $4 \mathrm{~F}$ or cosine $4 \mathrm{~F}$ terms means that our data contain neither the usual type of oblique anisotropy nor any anisotropy that is equivalent except for a rotation of the data within the plane of Figure 1. To a first approximation, then, we can conclude that sensitivity to movement is independent of the movement's direction.

\section{EXPERIMENT 2}

\section{Method}

Having established the isotropy of motion sensitivity, we next sought to determine whether it would be possible to mask the moving target by broadband directional noise. The noise consisted of random oscillations of the dot pattern during the foreperiod, prior to the onset of unidirectional motion. As before, coordinates for a spatially random pattern were stored pairwise in a table, $X_{1}, Y_{1}, \ldots, Y_{512}$. In addition, a second table was also computed before the actual display was required, containing pairs of $X$ and $Y$ increments. These increments were computed by a routine that converted from polar to Cartesian coordinates. For each pair of increments, a pseudorandom number, $\theta$, ranging from 0 to $2 \pi$, was generated and passed as an argument to the conversion routine. The routine then computed $X_{\text {inc }}=A \cos (\theta)$ and $Y_{\text {inc }}$ $=A$ sine $(\theta)$ for each value of $X$ and $Y$ when $A$ is the amplitude of the displacement of the dots, relative to their original position at the start of the oscillations.

To produce the oscillation, before plotting on the CRT, an $X$ increment was added to the $X$-coordinate of each dot, and $a$ $Y$ increment to its $Y$-coordinate. In each frame, a single pair of $X$ and $Y$ increments were used to displace all of the dots; from frame to frame, this pair changed, being selected from the previously computed table of 500 increment pairs. Using this algorithm, the pattern was just as likely to jump in one direction as in any other. This was achieved by producing the increment table from random numbers uniformly distributed over the interval 0 to $2 \pi$. Note that increment pairs were always added to the original starting position of the dots. This caused the oscillations to be noncumulative; on any frame, the position of each dot was uncorrelated with its position on the preceding frame. The result of this noncumulative pattern of oscillations was a distribution of amplitudes ranging from $0.16^{\prime}$ to $19.27^{\prime}$, with an average jump of $12.18^{\prime}$. At a frame rate of $33 \mathrm{~Hz}$, these correspond to velocities of $.09^{\circ} / \mathrm{sec}, 10.6^{\circ} / \mathrm{sec}$, and $6.7^{\circ} / \mathrm{sec}$, respectively.
A sufficient number of oscillation increments was computed and stored to ensure that on any trials the oscillations would form a nonrepeating sequence. In addition, the pattern of oscillations was varied between trials by entering the stored set of increments at a randomly chosen location. Finally, for each experimental session, an entirely new random sequence of oscillation increments was computed.

During the oscillation period, from frame to frame, the dots moved randomly in any direction. These movements maintained the spatial phase relationships among elements in the pattern, and caused the pattern to appear as though the dot display were being rapidly shaken in all directions in a plane perpendicular to the observer's line of sight. Direction and amplitude distributions of oscillatory movement averaged over five trials are shown in Figure 2.

The data shown in Figure 2 were collected by calculating the actual pattern of oscillation which the dots underwent on five randomly selected trials. For these calculations, foreperiod durations were assumed equal to their average value over all trials, $2.75 \mathrm{sec}$. From Figure $2 \mathrm{a}$ it can be seen that, on the average, each direction was equally represented. In Figure $2 b$, the dis-
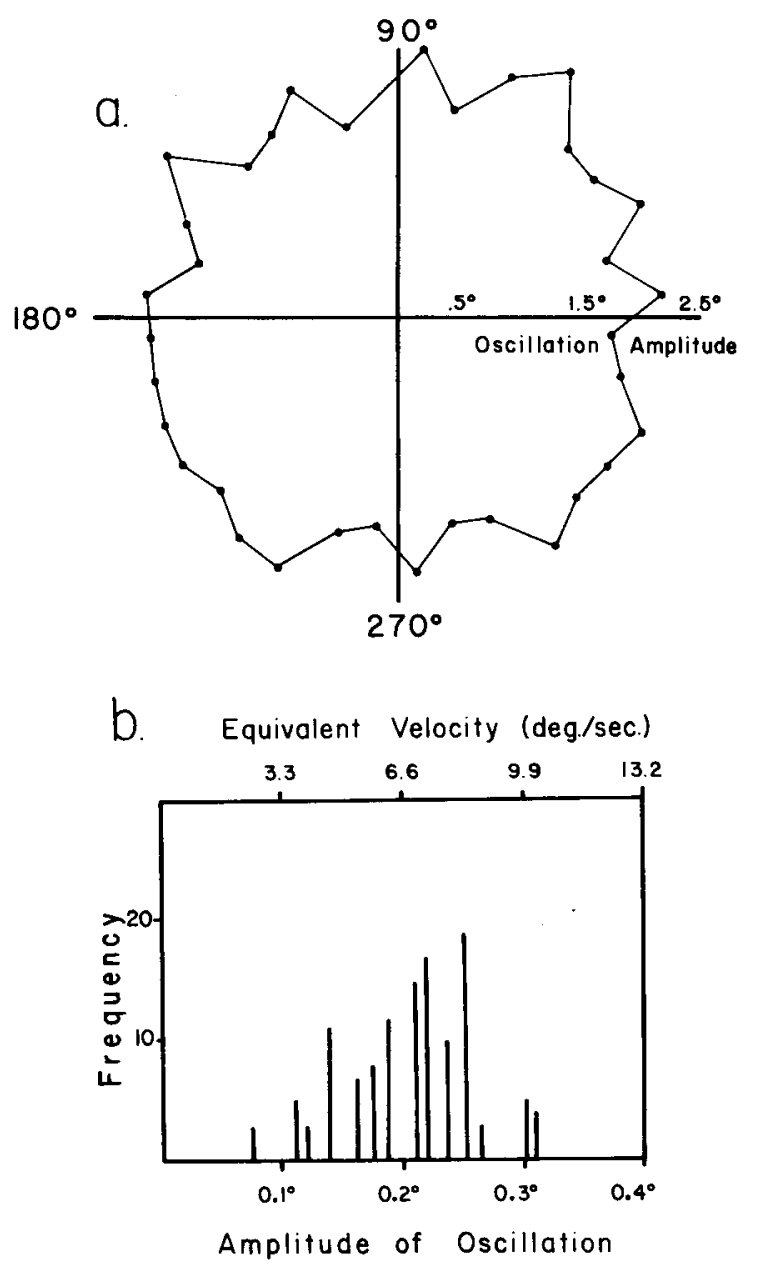

Figure 2. Amplitude and direction characteristics of broadband visual noise. (a) Total trialwise oscillation amplitude in various directions, averaged over five trials. (b) Frequency of oscillations of various amplitudes in degrees per second (lower abscissa). Equivalent velocities are calculated by assuming that a single, measured oscillation was to be repeated each display frame for an entire second. 
tribution of various amplitudes of movement is shown for five trials. Note that the mean amplitude of oscillation was slightly greater than, but within one standard deviation of, the speed $\left(5.3^{\circ} / \mathrm{sec}\right)$ of the unidirectional test movement.

Reaction times to eight different directions of motion were measured following random foreperiods of 2.0-3.5 sec, containing either oscillating noise or stationary patterns. The test directions, constant over a block of 50 trials, were $0^{\circ}, 45^{\circ}, 90^{\circ}, 135^{\circ}, 180^{\circ}$, $225^{\circ}, 270^{\circ}$ and $315^{\circ}$. Four observers (K.B., S.L., P.T., and J.C.) served in two 50-trial blocks per test direction. Each had normal or corrected-to-normal vision. K.B. and S. L. had also served in Experiment 1; P.T. and J.C. were inexperienced in this type of experiment.

\section{Results and Discussion}

Again, reaction times of less than $100 \mathrm{msec}$ or greater than $600 \mathrm{msec}$ were censored prior to data analysis. Figure 3 shows the mean for all observers of the geometric means for each test direction with a stationary foreperiod pattern and with a randomly oscillating foreperiod pattern. The inner circle indicates the overall mean with stationary foreperiod $(256.1 \mathrm{msec})$; the outer circle indicates the overall mean $(310.8 \mathrm{msec})$ wth oscillating foreperiod. The oscillations in the foreperiod increase reaction times by an average of $50.7 \mathrm{msec}$.

An analysis of variance on these data showed a significant effect of type of foreperiod stimulus $[F(1,3)=177.76, p<.001]$. Neither the direction of test motion nor the interaction between test direction and foreperiod type were significant sources of variance $(F=2.41$ and 1.25 , both $p>.05)$. The nonsignificant interaction reflects the fact that the broadband foreperiod noise had its desired effect: it uniformly reduced the visibility of all test directions.

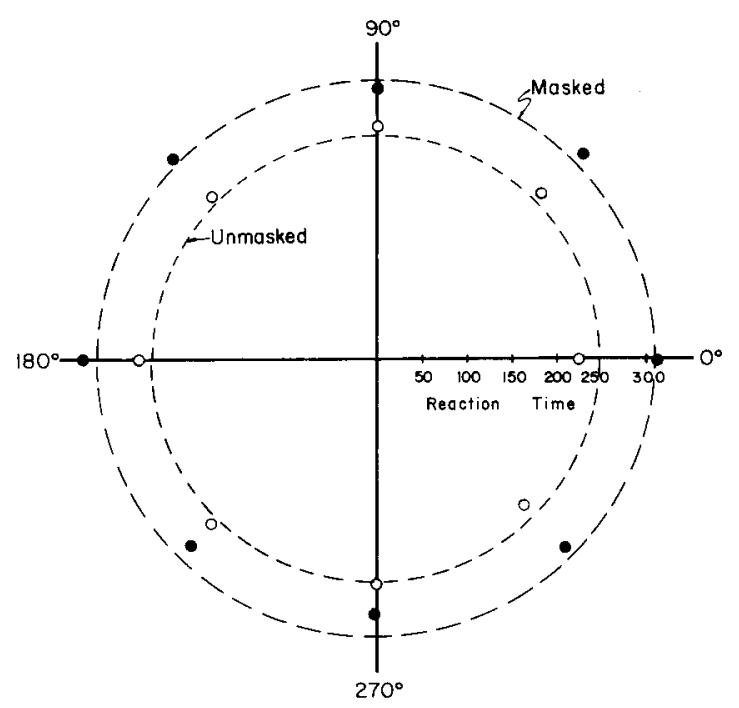

Figure 3. Mean reaction time for each test direction following stationary foreperiod stimulus (inner, unfilled circles) and with randomly oscillating foreperiod stimulus (outer, filled circles). Inner and outer dotted circles indicate grand means for stationary pattern and broadband noise foreperiod trials, respectively.
The lack of a significant main effect for test direction confirms the isotropy found in Experiment 1.

\section{EXPERIMENT 3}

Having demonstrated the potency of randomly oscillating noise for affecting the reaction to unidirectional motion, we next wanted to determine how the detectability of unidirectional motion would be altered if the observer had to discriminate it from noise that had been filtered to eliminate certain directional components. In the domain of spatial frequency, Stromeyer and Julesz (1972) found that only noise within a limited band around a test grating frequency could mask the grating. We wondered whether a similar result might hold for visual motion. Would the detectability of upward motion, for example, be affected only by the components of the oscillating pattern within the upward vicinity? This question has important theoretical implications, since an affirmative answer would permit us to apply the concept of a critical band (Fletcher, 1940) or tuned filter to the study of visual motion.

\section{Method}

In order to verify that the noise in the foreperiod had a direct effect on visibility of motion per se, and did not increase reaction times by some irrelevant means such as increased distraction of the observer, we produced filtered noise which might affect RTs for some test directions, but not others. We modified the foreperiod noise presentation by eliminating oscillatory movements in successively wider bands around the $90^{\circ}-270^{\circ}$ axis. In this experiment, all oscillations in the noise were of equal amplitude $\left(9.64^{\prime}\right)$, the same framewise amplitude as that of the tobe-detected, upward movement.

Although all oscillations were the same size, we placed restrictions on the directions in which the dot pattern could oscillate. Instead of using random numbers in the range 0 to $2 \pi$ to compute the table of increment values, we replaced random numbers in the interval from $[(\pi / 2)-(\mathrm{k} / 2)]$ to $[(\pi / 2)+(\mathrm{k} / 2)]$ and from $[(3 \pi / 2)-(k / 2)]$ to $[(3 \pi / 2)+(k / 2)]$ with numbers from outside these intervals; $k$ is the width (in radians) of the band of directions in which no oscillation was permitted. This ensured that the dot pattern would oscillate randomly in all directions except those in the band around the vertical axis. By eliminating equal upward and downward vectors, the resultant stimulus has zero net drift over time. Various bands of movement were eliminated, producing six different stimuli with progressively greater amounts of noise filtered out around the vertical axis. For all conditions, the stimuli had the same total power; only the meridional distribution of that power varied. Each of these stimuli, as well as completely random broadband noise, was used as a foreperiod stimulus of oscillating spatially random dots.

On each trial, the observer saw one of the oscillating stimuli for 2.0-3.5 sec; then the pattern began to move upward at $5.3^{\circ} / \mathrm{sec}$. The observer had to depress the telegraph key as soon as the unidirectional movement began. As before, reaction times were measured to the nearest millisecond. In each block of 50 trials, 25 trials had broadband noise in the foreperiod and 25 trials had one of the six degrees of filtered noise in the foreperiod. This provided a way to compare, for each block of trials, RTs for the various filtered noise conditions with a baseline RT of random broadband noise. Two observers (K.B. and D.B.) served in four sessions with each type of foreperiod stimulus. Both observers had served in earlier experiments. 


\section{Results and Discussion}

The mean reaction times with broadband directional noise were 329.6 and $356.8 \mathrm{msec}$ for Observers D.B. and K.B. The corresponding standard errors were 4.7 and $6.7 \mathrm{msec}$. False alarms, or responses made in less than $100 \mathrm{msec}$, were tabulated for each observer in each condition. For Observer D.B., these premature responses occurred $3 \%$ of the time for both the filtered noise trials and the broadband noise trials. For Observer K.B., the corresponding rates were $2 \%$ and $3 \%$. RTs to upward motion in each of the six filter conditions were computed and expressed as ratios to their respective control RTs (RT to the broadband noise). These ratios were used in all subsequent analyses. Figures 4 and 5 (curves labeled "No Drift") show that as increasingly wider sets of noise were filtered from the broadband noise, RT to upward motion declined. An ANOVA indicated that this variation in RT was statistically significant $[F(6,6)=19.12, p<.01]$. Thus, the results indicate that as noise was removed along the vertical axis $\left(90^{\circ}-270^{\circ}\right)$, the masking ability of the noise was reduced.

Since our main interest was in mechanisms sensitive to direction of motion, we wondered whether the effect of filtered noise observed in Experiment 3 was merely axial or truly directional in nature. Recall that, in order to prevent overall pattern drift in Experiment 3, oscillatory foreperiod movement was simultaneously eliminated around both upward and downward directions. To determine if the visibility of our upward test motion was affected primarily by the upward components of the noise (i.e., a directional

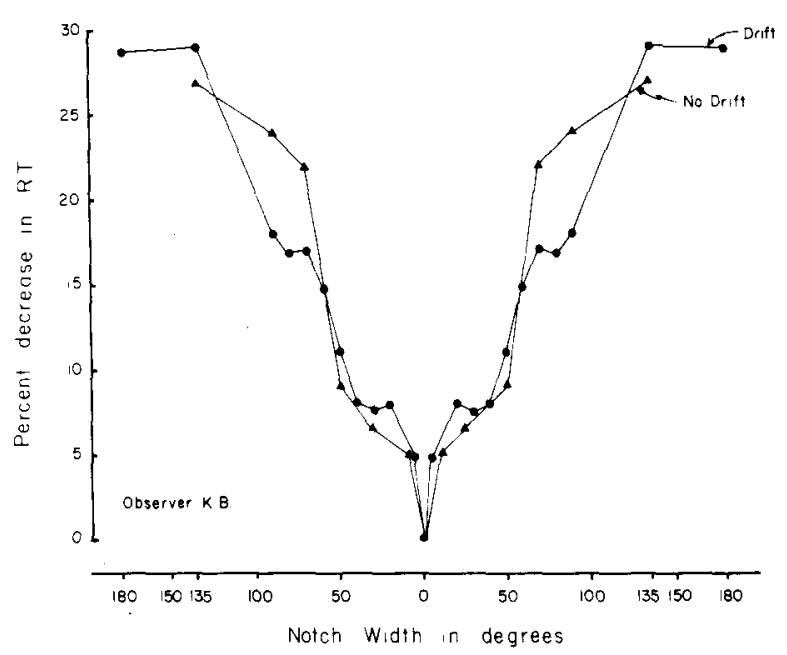

Figure 4. Data for observer K.B. from Experiments 4 and 5. The ordinate shows the percent decrease in RT compared to RT with broadband, nonfiltered noise. The abscissa shows the width of the band of noise which has been filtered out of the foreperiod stimulus. Curve labeled "No Drift" connects data from Experiment 4; curve labeled "Drift" connects data from Experiment 5.

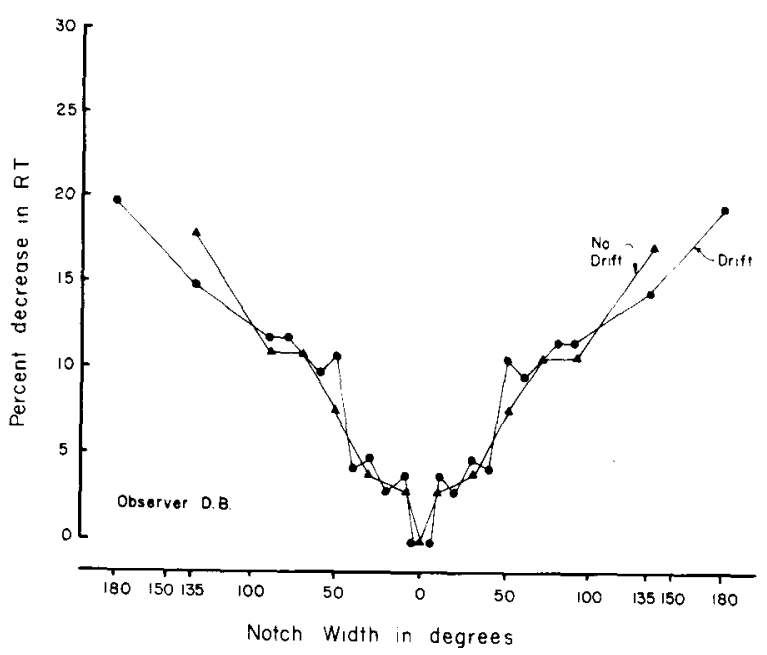

Figure 5. Data for Observer D.B. from Experiments 4 and 5. The ordinate shows the percent decrease in RT compared to RT with broadband, nonfiltered noise. The abscissa shows the width of the band of noise which has been filtered out of the foreperiod stimulus. Curve labeled "No Drift" connects data from Experiment 4; curve labeled "Drift" connects data from Experiment 5.

effect) or if it was affected by both upward and downward components (i.e., an axial effect), the following experiment was performed.

\section{EXPERIMENT 4}

If the effect observed in Experiment 3 were directional, then, by definition, only noise components proximate to upward would be effective maskers of the upward test motion; the presence of noise proximate to downward would be irrelevant as far as masking is concerned. However, if the effects observed in Experiment 3 were axial, then any noise components proximate to the up-down axis would contribute to the masking of the upward test motion; reducing either upward or downward noise components would reduce the masking. The noise in the present experiment differed from that of Experiment 3 only with regard to the differential presence of downward noise components. If the present experiment yielded the same masking of the upward test motion, we could conclude that the masking effect observed in Experiment 3 (and here as well) was directional rather than axial.

\section{Method}

We measured RTs to upward motion following broadband noise containing all directions as well as following 12 types of noise from which different amounts of upward motion had been filtered. From these stimuli were removed bands of noise, centered about upward, $5^{\circ}, 10^{\circ}, 20^{\circ}, 30^{\circ}, 40^{\circ}, 50^{\circ}, 60^{\circ} 70^{\circ}, 80^{\circ}, 90^{\circ}$, $135^{\circ}$, or $180^{\circ}$ wide. This large number of filtered stimulus types was designed to give a precise definition of the tuning of mechanisms sensitive to upward movement. 
As mentioned earlier, one consequence of filtering only noise proximate to one direction (upward) is a steady drift in the opposite direction (downward). The average rate of downward drift varied with the width of the band of noise filtered out of the masking stimulus. As the filtered band increased from $5^{\circ}$ through $180^{\circ}$, the resultant drift rate increased to $.16^{\circ}, .32^{\circ}, .59^{\circ}, .91^{\circ}$, $1.17^{\circ}, 1.44^{\circ}, 1.71^{\circ}, 1.92^{\circ}, 2.19^{\circ}, 2.40^{\circ}, 3.14^{\circ}$, and $3.41^{\circ} / \mathrm{sec}$.

With the exception of a greater number of different foreperiod stimuli, Experiment 4 was identical to Experiment 3 in all respects, including the use of the same observers, K.B. and D.B. Of course, as already indicated, noise in the downward direction was not filtered out.

\section{Results and Discussion}

The mean reaction times with broadband directional noise were 333.0 and $351.4 \mathrm{msec}$ for Observers D.B. and K.B.; associated standard errors were 5.5 and $5.8 \mathrm{msec}$. False alarms, i.e., premature response, were again tabulated for both observers. For D.B., the rates were $3.5 \%$ and $4.3 \%$, and for K.B., $3 \%$ and $3 \%$, for the filtered noise trials and broadband noise trials, respectively. As before, the RT to upward motion in each condition of filtered noise was computed and expressed in a ratio to the corresponding reaction time following random, broadband noise. These ratios were used in all subsequent analyses.

The results for the two observers can be seen in Figures 4 and 5 (curves labeled "Drift"). The variation in RT to upward motion with various bands of upward noise removed is statistically significant $[F(11,11)=7.58, p<.001]$ and is of the same magnitude as that found previously, in Experiment 3. In fact, the results are strikingly similar to those obtained in Experiment 3 (curves in Figures 4 and 5 labeled "No Drift").

The curves in Figure 4 are so similar that, within measurement error, we may consider them identical. The same is true for the curves in Figure 5. But the similarity is not so impressive if one compares curves between the two figures, that is, if one compares the tuning functions for the two observers. Although we cannot verify the interobserver differences statistically, it does appear that directional tuning is sharper for K.B., the far more experienced observer, than for D.B. In particular, as an increasing amount of noise was filtered from the masking stimulus, K.B.'s reaction times changed more rapidly than did D.B.'s. As a result of these apparent differences between observers, we performed another experiment, designed to explore the possibility of stable betweenobserver differences in directional selectivity.

\section{EXPERIMENT 5}

Suppose the RT functions in Figures 4 and 5 reflect the directional selectivity of upward-sensitive visual mechanisms, and suppose that K.B. actually does exhibit sharper tuning than D.B. This difference between observers should then also show up in any other task which depends upon the selectivity of these same mechanisms. Since directional selectivity should be straight forwardly related to the ability to recognize small differences in direction of motion, Experiment 5 compared the difference threshold for direction of motion for Observers D.B. and K.B. Consistent with the tuning differences observed in the previous experiment, we expected D.B. to have a larger difference threshold than that for K.B.

\section{Method}

This experiment employed the method of constant stimuli. Each trial consisted of two 600 -msec intervals separated by $1 \mathrm{sec}$. In the first interval, the standard stimulus (upward movement) was presented; in the second interval, the random dot pattern either moved upward again, or $1^{\circ}$ or $2^{\circ}$ clockwise or counterclockwise relative to the standard. Following each trial, the observer's task was to indicate whether the movement in the second interval appeared clockwise or counterclockwise, relative to the upward movement in the first interval. The five possible directions which could occur in the second interval were randomized, and 100 trials (20 of each direction) were run per block.

\section{Results and Discussion}

The percentage of "clockwise" judgments were tabulated for each observer and condition. Figure 6 shows the $\mathrm{z}$ scores corresponding to these percent "clockwise" judgments plotted against the difference, in degrees, between the two directions of motion. The lines shown in that figure are the best fitting lines in the least squares sense. For each observer, a curve was fit, using the estimates from three different sessions for each point plotted in Figure 6. The fitted lines account for $92 \%$ (Observer K.B.) and $86 \%$ (Observer D.B.) of the total variance in the data. To estimate the statistical reliability of dif-

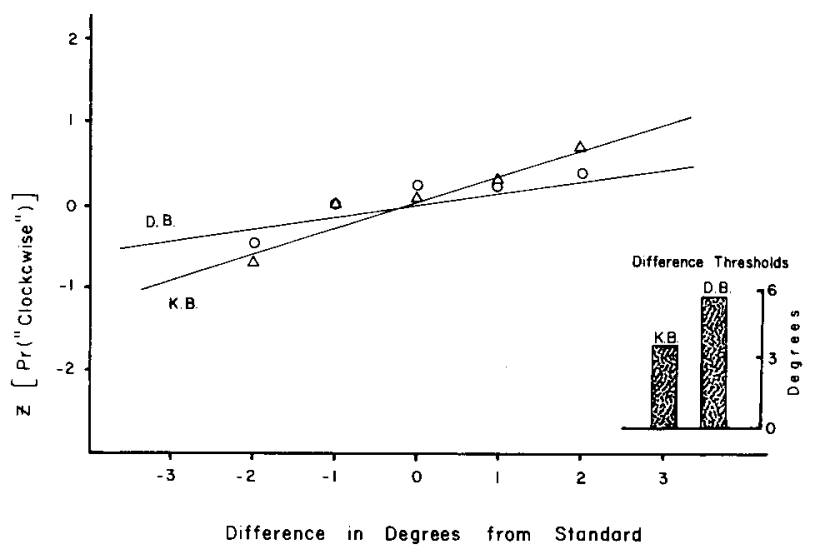

Figure 6. Percent "clockwise" judgments as a function of the difference in degrees between two directions of motion presented on each trial. Abscissa values range from negative, counterclockwise, stimuli to positive, clockwise, stimuli to linearize the data. Ordinate values are given as $z$ scores; all curve fitting and calculations were done with the $z$ scores. Shown are best fitting lines for Observers D.B. and K.B. The insert shows directional difference thresholds, in degrees, for the two observers. 
ferences between the slopes for the two observers, we calculated $95 \%$ confidence limits. The slopes and associated confidence limits are $.30 \pm .08$ and $.12 \pm .05$ for Observers K.B. and D.B., respectively. The intervals defined by the confidence limits for the two observers are nonoverlapping. Difference thresholds, are approximately $3^{\circ}$ for Observer K.B. and $6^{\circ}$ for Observer D.B. This outcome is consistent with the individual differences observed in Experiment 4: the observer with the broader tuning functions (D.B.) also shows less precision in judging direction of motion.

\section{GENERAL DISCUSSION}

Readers familiar with the literature on reaction times may have noted that even the shortest times reported for our experiments are approximately $30-40$ msec longer than reaction times which might be expected for alert and highly motivated observers responding to intense signals (e.g., Teichner \& Krebs, 1972). We believe that two factors account for the longer than expected times in our experiments. First, the target speed to which we recorded reaction times, $5.3^{\circ} / \mathrm{sec}$, yields slower reaction times than other, faster speeds of movement we have worked with in other experiments. For example, in related work, we've found average, unmasked reaction times of $210 \mathrm{msec}$ to the $5.3 \% \mathrm{sec}$ movement, but RTs $10 \mathrm{msec}$ faster to $16^{\circ} / \mathrm{sec}$ movement. But there is another, more significant, cause for our extended RTs. Note that our computer measured response latency from the instant when the first dot was plotted in the first frame of unidirectional motion. From the observer's point of view, however, the very first sign that the unidirectional motion had begun was at least a full display frame $(33 \mathrm{msec})$ away. Theoretically, some dots in the second frame of movement would have had to be plotted before motion could possibly have been detected. At a minimum the detection of unidirectional motion requires the detection of the correlation between dots plotted in successive frames. So, although our computer routines began keeping time when the computer began to produce movement, the observer couldn't possibly have detected it until a minimum of another $33 \mathrm{msec}$ had elapsed. Together, the two factors mentioned here account for the somewhat longer than usual RTs we obtained. Rather than try empirically to determine the point in the stimulus presentation which was the effective start of motion, we thought it best to begin our latency measure relative to the stimulus nominal start. The net result is that a constant of at least $33 \mathrm{msec}$ is added to all our RTs.

It should be noted that the observer's uncertainty about the time of onset of motion (temporal uncertainty) would have elevated RTs in our experi- ments by some small constant. But, since this uncertainty was equally present in all conditions, it cannot play a role in our results, where comparisons are always made between masked and unmasked RTs.

Our use of RTs to measure visibility requires some additional comment. As previously indicated, RT is an attractive dependent variable because it bridges the domain of small signals, indexed by standard threshold measures, and the domain of the more intense signals of everyday life (Green \& Swets, 1966). Depending on the assumptions one is prepared to make, RT functions like those of Figures 4 and 5 can be made to support theoretical statements of various weights. Visibility, which we used RT to measure, is most commonly defined by the reciprocal of the stimulus energy required to produce some criterion performance level. Thus, a stimulus is said to be more visible than another if less energy is required to reach the criterion performance level. The weakest assumption we are prepared to make is that RT is a nonincreasing function of target visibility. This assumption is supported by experiments in which stimulus energy is varied and RTs obtained (e.g., Teichner \& Krebs, 1972; Ueno, 1977). With this weak assumption, the functions of Figures 4 and 5 show that noise components, even those more than $45^{\circ}$ away (i.e., filter band $90^{\circ}$ wide), can affect the visibility of upward motion. As we shall see later, such directional selectivity is comparatively poor. A considerably stronger assumption is that RT varies linearly with visibility. Although already existing data (e.g., Teichner \& Krebs, 1972) clearly contradict claims for the linearity of RT over a large range of visibilities, the small variation in RT observed in our experiments virtually guarantees the triviality of departures from linearity over our quite limited range. Certainly, a linear approximation would likely not distort the underlying function very much when, at most, RTs vary no more than $30 \%$. With the assumption of approximate linearity, Figures 4 and 5 suggest that not only are there interactions between test and masking components separated by $45^{\circ}$, but that these interactions are substantial: at a filter width of $90^{\circ}$, nearly half of the total directional masking effect still remains.

Still other assumptions would be required in order to derive a more precise estimate of the bandwidth of visual mechanisms sensitive to upward motion. The most important of these assumptions would involve the shape of the mechanism's sensitivity profile. A good discussion of these assumptions is provided by Patterson (1974).

We have previously considered the claim that our masking effects are directional rather than axial. Here we would like to offer one additional bit of evidence to support our interpretation. In Experiments 3 and 4, RTs were measured for a constant 
direction of motion, upward, under conditions in which the directional distribution of noise was varied. In experiments reported elsewhere (Ball \& Sekuler, Note 1), we have done the logical converse: we measured RTs to various directions of test motion following exposure to a constant filtered noise masker. In one of those experiments, the masking noise was filtered to remove directional components in a band of $45^{\circ}$ on either side of upward. Although RTs to upward test motion were virtually unaffected by the filtered noise, RTs to downward motion were elevated by an average of $15 \%$. Note that both test directions fall along the same axis. Of course, this also increases our confidence that the effects reported here, for Experiments 3 and 4, are directional rather than axial. Since control measures indicate that unmasked RTs to upward and downward are equal, the differential masking of upward and downward clearing motion is a directional rather than an axial effect.

Having established that the masking effects reported earlier are directional in character, we can now try to compare the directional selectivity we observed with previous measures of selectivity. Using random dot patterns, Levinson and Sekuler (1976) estimated the tuning of direction-specific mechanisms by finding the perceived direction shift for test motion as a function of the adapting direction. Using this procedure, they, too, found rather broad tuning. Even with as much as $60^{\circ}$ difference between the directions of adapting and test dots, preexposure to moving adaptation dots could substantially alter the direction in which the test dots appeared to move. Börjesson (Note 2) measured the perceived direction of the motion aftereffect and also found similarly broad tuning. Generally, then, our tuning functions agree with these previous results. Although differences among response metrics make direct, detailed comparisons of tuning difficult, all the studies, including our own, indicate that visual mechanisms responsible for the perception of motion are very broadly tuned along the direction axis: A mechanism has appreciable sensitivity to movement $45^{\circ}$ or more away from its optimal direction.

It is also instructive to compare our measurements to those which have been made on orientation selectivity. Campbell and Kulikowski (1966) reported that at $12^{\circ}$ on either side of a vertical test grating, their masking effect was reduced by a factor of two with respect to its maximum value. Others, using a variety of techniques estimated that the human orientation units have effective half-widths of $8^{\circ}-12^{\circ}$ (Campbell \& Maffei, 1970). In our studies, the masking effect was reduced by a factor of two at approximately $45-50^{\circ}$ on either side of the vertically moving stimulus.

In this paper we have called attention to the parallels between measures of directional selectivity defined by masking experiments and the corresponding size of difference thresholds. These parallels also extend to the domain of orientation. For example, as we have just seen, orientation tuning functions are narrower than those for direction of motion. Even the more precise of our two observers had a direction difference threshold more than 10 times larger than previously reported difference thresholds ior orientation (Sekuler, Rubin, \& Cushman, 1968). The parallels we have identified suggest that it wiil bo worthwhile in the future to consider the extent to which tuning and discrimination measures may covary in other conditions as well.

We have one final point, concerning betweenobserver differences in our experiments. Experiments 3 and 4 showed that Observer K.B. had a narrower directional tuning function than did $\mathrm{Ob}$ server D.B. Experiment 5 showed that directional difference thresholds for the two observers not only differed significantly, but did so in a way consistent with the hypothesis that Observer K.B.'s upwardsensitive mechanism was more sharply tuned than D.B.'s. One obvious source of stable individual differences is differential practice with the experimental task or other, related tasks. Recently, for example, DeValois (1977) has shown that repeated measurements over a year alter the spatial contrast-sensitivity function by as much as $.8 \mathrm{log}$ unit. In addition, DeValois noted that over time the threshold elevation produced by a sinusoidal grating became more sharply tuned for spatial frequency. In our work, Observer K.B., who showed the better discrimination and narrower tuning, had had approximately $70 \mathrm{~h}$ of practice on related tasks before beginning the experiments reported here; Observer D.B. had had only 10 hours practice. We believe that visual psychophysics is a sufficiently mature science, that it must begin to confront the existence of stable differences between the capacities and characteristics of various normal observers. We are currently exploring the effect of practice on the detection of motion in the presence of filtered noise as well as its effect on direction difference thresholds.

\section{REFERENCE NOTE}

1. Ball, K., \& Sekuler, R. Directional selectivity: Tuning functions and discrimination performance. Paper presented at the meeting of the Psychonomic Society, Washington, D.C., November 1977 .

2. Börjesson, E. Perceived direction of motion aftereffects. Department of Psychology, University of Upsalla, Sweden, Report $188,1976$.

\section{REFERENCES}

Appelle, S. Perception and discrimination as a function of stimulus orientation: The "oblique effect" in man and animals. Psychological Bulletin, 1972, 78, 266-278. 
Campbell, F. W., \& Kul.1kowski, J. J. Orientational selectivity of the human visual system. Journal of Physiology, 1966, 187, 437-445.

Camprell, F. W., \& Maffei, L. Electrophysiological evidence for the existence of orientation and size detectors in the human visual system. Journal of Physiology, 1970, 207, 635-652.

DEV ALoIs, K. Spatial frequency adaptation can enhance contrast sensitivity. Vision Research, 1977, 17, 1057-1065.

Fletcher, H. Auditory patterns. Reviews of Modern Physics, 1940, 12, 47-61.

Green, D. M., \& Swets, J. A. Signal detection theory and psychophysics. New York: Wiley, 1966.

Keck, M. H., Pallela, T. D., \& Pantle, A. Motion aftereffect as a function of the contrast of sinusoidal gratings. Vision Research, 1976, 16, 187-191.

Levinson, E., \& Sekuler, R. The independence of channels in human vision selective for direction of movement. Journal of Physiology, 1975, 250, 347-366.

Levinson, E., \& Sekuler, R. Adaptation alters perceived direction of motion. Vision Research, 1976, 16, 779-781.

Lipson, S. G. Optical transforms in teaching. In H. Lipson (Ed.), Optical transforms. New York: Academic Press, 1972.

Patterson, R. D. Auditory filter shape. Journal of the Acoustical Society of America, 1974, 55, 802-809.

Patterson, R. D. Auditory filter shapes derived with noise stimuli. Journal of the Acoustical Society of America, 1976, 59, 640-654.
Sekuler, R. Visual motion perception. In E. Carterette \& M. Friedman (Eds.), Handbook of perception (Vol. 5). New York: Academic Press, 1975.

Se kule R, R., \& Armstrong, R. Fourier analysis of polar coordinate data in visual physiology and psychophysics. Behavior Research Methods \& Instrumentation, 1978, 10, 8-14.

Sekuler, R., \& BalL, K. Mental set alters visibility of moving targets. Science, 1977, 198, 60-62.

Sekule r, R., \& Ganz, L. Aftereffect of seen motion with a stabilized retinal image. Science, 1963, 139, 419-420.

Sekuler, R., Rubin, E. L., \& Cushman, W. H. Selectivities of human visual mechanisms for direction of movement and contour orientation. Journal of the Optical Society of America, 1968, 58, 1146-1150.

Stromeyer, C. F., III, \& Julesz, B. Spatial-frequency masking in vision: Critical bands and spread of masking. Journal of the Optical Society of America, 1972, 62, 1211-1232.

Teichner, W. H., \& KreBs, M. J. Laws of the simple visual reaction time. Psychological Review, 1972, 79, 344-358.

UENo, T. Reaction time as a measure of temporal summation at suprathreshold levels. Vision Research, 1977, 17, 227-232.

(Received for publication February 8, 1979; revision accepted June $20,1979$. 